Man and Nature

L'homme et la nature

\title{
Un Philosophe contre la cour : le marquis d'Argenson et l'échec de la réforme politique sous Louis XV
}

\section{Neal Johnson}

Volume 10, 1991

URI : https://id.erudit.org/iderudit/1012624ar

DOI : https://doi.org/10.7202/1012624ar

Aller au sommaire du numéro

Éditeur(s)

Canadian Society for Eighteenth-Century Studies / Société canadienne d'étude du dix-huitième siècle

ISSN

0824-3298 (imprimé)

1927-8810 (numérique)

Découvrir la revue

Citer cet article

Johnson, N. (1991). Un Philosophe contre la cour : le marquis d'Argenson et l'échec de la réforme politique sous Louis XV. Man and Nature / L'homme et la nature, 10, 67-75. https://doi.org/10.7202/1012624ar

Copyright (c) Canadian Society for Eighteenth-Century Studies / Sociéte canadienne d'étude du dix-huitième siècle, 1991
Ce document est protégé par la loi sur le droit d'auteur. L'utilisation des services d'Érudit (y compris la reproduction) est assujettie à sa politique d'utilisation que vous pouvez consulter en ligne.

https://apropos.erudit.org/fr/usagers/politique-dutilisation/ 


\section{Un Philosophe contre la cour : le marquis d'Argenson et l'échec de la réforme politique sous Louis $\mathrm{XV}$}

René-Louis d'Argenson a eu toute sa vie le sentiment d'être seul contre tous, de prêcher dans le désert, d'ignorer totalement l'art de se faire écouter, talent possédé au plus haut degré par le plus médiocre de ses adversaires. Réformateur infatigable, il n'a rien pu réformer. Travailleur inlassable, il est mort littéralement la plume à la main, laissant une oeuvre immense, dont presque rien pourtant ne fut publié de son vivant. On connaît, depuis quelque temps, dans des éditions plus ou moins fidèles, son Journal, ses Considérations sur l'état ancien et nouveau de la France, ses Loisirs d'un ministre et ses Notices sur le théâtre. Mais aujourd'hui, grâce aux richesses des Archives d'Argenson déposées à l'Université de Poitiers, nous disposons d'un nombre impressionnant de textes inédits qu'on avait cru perdus: 13 mémoires autographes sur les affaires du temps, un Journal de campagne, des Pensées sur la Réformation del'Etat (PRE) en 150 articles, des Pensées diverses sur la politique (PDP) en 96 articles, des Remarques en lisant (REL) en 1000 articles, ainsi que de sa correspondance avec son frère cadet, le comte Marc-Pierre d'Argenson, ministre de la guerre de 1742 à 1757 . A travers toute cette oeuvre, il est clair que, jusqu'à sa mort en 1757, le marquis resta persuadé, contre toutes les apparences, que le travail et le mérite personnel finiraient par triompher des égoïsmes, des cabales, des privilèges, et de la vanité d'esprits médiocres et intéressés. Il ne perdit jamais l'espoir que la confiance du monarque (condition nécessaire à toute tentative de réforme) viendrait un jour récompenser la compétence, l'effort et la vertu. A la fin, certes, il dut se plier à l'évidence que tel fut rarement le cas dans la France de Louis XV; il ne désespéra pourtant jamais de pouvoir servir sa patrie, non seulement en espérant qu'on le rappelle au pouvoir, mais aussi en sollicitant activement (et sans succès) des emplois, même des plus modestes.

Sa courte carrière publique fut indéniablement non seulement un échec lamentable, mais une humiliation personnelle. D'Argenson se considérait le porte-parole d'une vertu civique romaine qui n'était guère à la modeà la cour de Louis XV. Il fut ambitieux, certes, ne cachant jamais 
sa volonté d'accéder au pouvoir, d'être nommé un jour premier ministre ou contrôleur-général. Cette ambition (ce rêve) revient si souvent sous sa plume qu'on peut même parler d'obsession. Mais on ne saurait mettre en doute sa sincérité quand il affirme que c'est pour mieux servir son roi et son royaume dans la meilleure tradition de sa famille, réputée depuis des générations pour son dévouement au bien public et son désintéressement.

Le marquis ne fut jamais premier ministre; son passage au ministère des affaires étrangères (1744-47) fut de courte durée et peu glorieux. Il fut non seulement disgrâcié, mais - et c'est la pire des injures disgrâcié sans être exilé, puisque jugé inoffensif. Ses projets, à l'époque et depuis, ont en effet été jugés aussi chimériques que ceux de l'abbé de Saint-Pierre, son maître à penser au Club de l'Entresol.

Ce n'est pas notre propos ici de décider du bien-fondé des idées politiques du marquis. Au lieu d'analyser sa pensée, il s'agit plutôt de mieux comprendre les forces en jeu, les obstacles qui se présentaient à l'époque à un réformateur zélé. Les documents inédits qui se trouvent aux Archives d'Argenson à Poitiers, dont certains révèlent sans fard sa pensée la plus intime, permettent au chercheur de se mettre à la place du marquis dans une mesure jusqu'alors impossible.

Disons pourtant, en passant, que ses idées, peu orthodoxes pour l'époque il est vrai, sont loin de paraître si ridicules aujourd'hui. En matière de science économique, c'est à lui qu'on doit le terme laissezfaire. Parmi ses prédictions on peut citer le canal de Suez, les ballons qui permettraient aux hommes de voler dans les airs avant la fin du siècle, la guerre d'indépendance des colonies britanniques en Amérique, et même une révolution en France. Il connaissait bien la campagne, et fut touché par la misère des paysans. En matière de politique, il était contre les privilèges de sa propre caste, pour l'abolition des 3 ordres, pour une égalité de droit, insistant pour que l'égalité résulte des différences des seuls talents, de sorte que chacun soit fils de ses oeuvres et de ses mérites, non de sa naissance. ${ }^{1}$ Que ce soit donc dit en passant, cette année du bicentenaire de la Révolution française, combien le marquis se serait trouvé à sa place le soir du 4 août 1789 .

Il faut ajouter qu'il est sans doute aussi vrai que son échec est dû en partie à certaines carences de sa pensée. On sait qu'il n'a pas compris le rôle que commençait à jouer la Prusse de Frédéric le Grand, nation qui sera pendant deux siècles l'ennemi héréditaire de la France. A sa décharge il conviendrait d'ajouter aussi, qu'à l'époque, ses compatriotes n'y voyaient pas plus clair que lui.

Mais au lieu de nous lancer dans ce genre de débat, nous voulons donc nous mettre à la place du marquis et voir sa lutte à travers ses propres yeux. On remarque d'abord son admiration pour un certain 
nombre de serviteurs fidèles de l'état qui, tout comme lui, avaient subi disgrâce ou persécutions injustes. Il évoque les cas du marquis de Fénélon, discrédité, perdu, chassé de son ambassade, tué misérablement à la guerre (REL 1207); du marquis de Pomponne, ministre de 1671 à 1689 , victime de l'inimitié de Louvois et de la rage de Mme de Montespan (REL 1251); du Maréchal de Bassompierre, victime de la jalousie de Richelieu, et qui passa 12 ans à la Bastille, et du comte de La Bourdonnais, grand capitaine, mais victime innocente des intrigues de rivaux jaloux. On trouve la même admiration pour ceux qui se sont tués à la tâche, ceux qui, refusant de se ménager, sont morts comme lui, la plume à la main. Dans cette catégorie il faut citer le grand érudit du Cange. ${ }^{2}$ Le cas du chancelier Bacon provoque à double titre l'admiration du marquis: manipulé par des favoris, dégradé, déshonoré, bouc émissaire des extorsions de Jacques Ier et de Buckingham; Bacon eut en plus le mérite de se tuer à force de travail.

L'admiration du marquis pour les serviteurs loyaux mais malheureux allait de pair avec un parfait mépris de ceux qui, sans talent, réussissent grâce à leurs protections. Il est vrai que ses ennemis personnels sont les premières victimes de sa plume. Quand il déclare notamment 'qu'aujourd'hui (au ministère) les esprits sont gauches et ignorants; avec des Maurepas, des Noailles, des c(omte) d'Argenson, il faut qu'un état périsse, ${ }^{3}$ il est sans aucun doute en train de régler des comptes à sa façon, c'est-à-dire en exorcisant les diables par des traits de plume dans des mémoires que personne ne lira. Mais on aurait tort d'attribuer ses réactions, parfois violentes, à sa seule rancune. Son acharnement contre le gouverneur de Niort, Michel-Ange de Castellane, ${ }^{4}$ par exemple, paraît réellement inspiré par la totale médiocrité du personnage.

De toute façon, aux yeux du marquis, se dressait contre lui une foule d'adversaires: toute une société corrompue prête à résister, pour des motifs non-avouables. Il y avait d'abord le vieux cardinal de Fleury, premier ministre depuis toujours et jusqu'en 1743, louable pour son désintéressement et son amour de la paix, mais à la fin de sa vie 'un vieux fol, un vieux tyran, un vieux singe,' se laissant facilement mener, qui, malgré ses bonnes intentions, eut le tort de donner sa confiance à la clique Maurepas-Tencin-Amelot, et au comte d'Argenson.

Plus tard un second obstacle, plus imprévisible puisque caché et changeant, fut la favorite, Mme de Pompadour, qui, devenue intendante des amusements du roi, 'a le premier suffrage et décide le plus fréquemment, mais non toujours, car souvent d'autres conseillers la contredisent, et le moindre rhumb de vent fait pirouetter la girouette, tant elle est flexible, tant notre monarque a peu de décision dans le caractère, ou pour mieux dire, il n'en a précisément aucune. ${ }^{5}$ Elle fut la 
créature des frères Pâris qui l'avaient placée auprès du roi, avec le résultat que ce sont eux qui gouvernaient, formant ainsi, à leur tour, obstacle à toute tentative de réforme. Entre la Pompadour et les frères Pâris, nous dit d'Argenson, 'le roi se trouve garrotté... Sans le savoir, le roi se trouve prisonnier dans un système et un entêtement pareil où le Régent se trouva pour Law en 1719 et $1720 .^{\prime}$

$\mathrm{C}^{\prime}$ est le roi lui-même qui fut pendant longtemps le seul espoir du marquis. Dès la mort de Fleury, le jour où Louis XV lança son 'Messieurs, me voilà donc premier ministre,' tous les espoirs étaient permis. 'A chaque quart d'heure la réputation du roi se rétablit dans le public, écrit-il, et bientôt elle éclatera comme celle d'Henri IV' (PRE 644). On osait croire que Louis était en passe de devenir le grand roi dont la France avait besoin: '(J'ai en lui), nous dit d'Argenson, la foi d'Abraham ... Louis XV est chéri de son peuple sans lui avoir fait encore aucun bien... (il) aura les qualités de Henri IV et de Louis XIV sans avoir leurs défauts.' Pendant la campagne de 1743 il était encore permis d'écrire 'Nos princes font merveille à l'armée: aurions-nous un roi?' Plus tard pourtant l'enthousiasme s'estompe: 'Je ne veux pas qu'on dise du mal du roi, je veux le croire bon. Parfois on disculpe le roi, plus souvent on l'inculpe. Pour moi qui l'aime avec passion, je saisis avec avidité tout ce qui peut le blanchir et l'élever' (PRE, fragment P).

Comme tout bon Français de l'époque, d'Argenson avait donc un fond presque inépuisable de respect et $d^{\prime}$ amour pour son roi. Avec le passage du temps pourtant, les verbes au présent et au futur sont remplacés par des conditionnels: 'Le Roi aurait un beau rôle à jouer, ce serait de se mettre à la tête de l'opinion et d'opérer lui-même les réformes. ${ }^{7}$ Sans oser se l'avouer, on ne croit plus que le roi passera à l'action; ${ }^{8}$ la girouette ne cessera jamais de tourner avec le vent. Le marquis est obligé de constater 'que le respect des rois se tourne en mépris chaque jour. Ainsi l'on n'est plus en respect que du nom, l'adoration est passée. Cela aura des suites' (PRE 703). Les jugements deviennent progressivement plus sévères: 'Louis $X V$ a trouvé l'étiquette et la magnificence établies par Louis XIV. Il eût cru déroger en retranchant le moindre article...A cela se sont joints l'apathie, l'indolence, le laisseraller. Puis une maîtresse, une courtisane, véritable fille entretenue, de celles qui exaltent la gloire d'un homme qui dépense beaucoup près des femmes. Car de son naturel, le roi n'est ni prodigue, ni magnifique, ni dénué de sagesse. Mais il est facile, léger et mou...Une telle conduite mène droit à l'anarchie. Nulle fermeté, nulle résolution, nulle décision quelconque. C'est la girouette sur laquelle soufflent chacun des courtisans qui l'entourent' (PDP 53, sept. 1751). Doux, sensible, bien intentionné, mais entouré de fripons (PRE 798), Louis XV dépense autant que 
ses prédécesseurs en bâtiments mais ce n'est ni par calcul, ni par orgueil, mais par mollesse. ${ }^{9}$

Parmi les 'fripons' qui constituaient l'entourage du roi, il faut signaler les jésuites. Malgré ses études au lycée Louis-le-Grand, où il était camarade de classe de Voltaire, le marquis condamne 'le vice général de leur société,' leur tolérance de l'idolâtrie, et leurs restrictions mentales (PDP, art. 15). Mais il y voyait surtout le symptôme d'une décadence s'étant installée en France depuis les deux reines Medicis et le cardinal Mazarin. Les jésuites symbolisaient donc la duplicité; pour le marquis, les jésuites, la cour et l'Académie française répandaient la même maladie: 'on disait l'autre jour qu'il fallait regarder les jésuites comme des missionnaires destinés à faire perdre à nos Français toute l'ancienne franchise gauloise...de sorte que tous nos jeunes et vieux courtisans ne sont plus aujourd'hui que de petits jésuites' (PDP, art. 28).

Il faudrait placer aussi dans le camp ennemi sa propre épouse, celle que le marquis appelait 'ma triste femme, (qui) ne marchait, ce sont ses propres paroles, que pour l'argent, me tourmentait, me ruinait pour avoir de l'argent, me haïssait' (PRE 680-681). Il alla jusqu'à demander une lettre de cachet contre elle dans une lettre à son frère le ministre: 'Mon cher frère, si vous n'obtenez pas incessament un ordre du Roy pour enfermer votre belle soeur, elle va commencer contre moi un procès fort ridicule; elle propose de faire imprimer le factum le plus injurieux et d'en envoyer à toutes les portes... Je demanderais qu'elle fût renfermée dans un couvent à 10 lieues de Paris, qu'elle fût enlevée à l'improviste, qu'on saisît ses papiers... (que) ses biens (soient) sequestrés chez un notaire..... ${ }^{10}$

Mais l'obstacle principal à sa carrière - et à tout programme de réforme en France, car les deux vont de pair dans l'esprit du marquis c'était bien la cour. Le marquis y était détesté par tous les courtisans qui lui trouvaient l'air ridicule et le surnommaient la bête. Collectivement ces courtisans (les Belle-Isle, Noailles, Puysieux) représentaient, aux yeux du marquis, la source des principaux maux dont souffrait le royaume. Si le roi était mal entouré, c'était la faute de la cour: 'On le trompe de tous cotés, il est entouré de favoris et de ministres plus contraires à son caractère qu'aucun de nos rois en ait ci-jamais été... Comment se choisissent les ministres...quand la cour influe sur tout ce qui se fait, quand des courtisans avides et écoutés décident du travail et du ministère? ${ }^{\prime 11}$ La mentalité du courtisan fourbe et intrigant est celle du joueur. Ecoutons encore d'Argenson: 'Le caractère du joueur forme celui du courtisan...qui rapporte tout à son profit, qui trouve des adversaires dans ses meilleurs amis...qui cherche la ruine de la société, qui aime le gros jeu parce qu'il cherche le profit plus quel'amusement, esprit petit mais exact recherchant les petits objets pour les grands, se vantant 
de perdre quand il gagne, préférant le lucre à la réputation, se riant du malheur et du désespoir des autres. ${ }^{12}$ 'La mauvaise chose qu' une cour, $s^{\prime}$ exclame le marquis, elle est cependant devenue le sénat de la nation. On y devient sénateur par les vices plutôt que par les vertus' (PRE 666). Il a rédigé un mémoire intitulé $L a$ Cour est le sénat et la cause destructive de la nation (déc. 1750), véritable profession de foi, où il déclare que 'les ministres sont devenus nécessairement courtisans, et s'ils ne le sont pas, ils sont promptement disgracié(s) comme corps étrangers et hétérogènes... Qu'y veut-on à la cour? Dépenser beaucoup pour le luxe, mépriser l'économie et la vertu, vendre les grâces, dispenser la loi, porter le prince aux faiblesses, s'opposer à toute réformation dans l'état. Ainsi plus il y aurait de besoin de cette réforme, plus elle est écartée par la cour. C'est la cour qui corrompt les moeurs de la nation par son luxe, sa dépense, ses faux-airs, son ignorance, et son intrigue au lieu d'émulation. Toutes les places, emplois et grades du militaire vont aux courtisans par préférence. De là il n'y a plus d'émulation de s'avancer par le mérite...Les jeunes gens savent en entrant dans le monde qu'on ne s'avance que par l'intrigue de cour, et négligent d'acquérir aucun mérite. On ne croit (pas) plus à la vertu qu'aux revenants. On s'accommode à la petite finesse jésuitique... Voilà les fruits de l'établissement qu'a fait Louis XIV d'une capitale à Versailles exprès pour la cour.'

Comme on peut donc le constater, pour le marquis, les structures de la société étaient fondamentalement viciées. Depuis un siècle, le royaume était atteint $d^{\prime}$ 'une souplesse italienne' dont, comme nous le verrons, sa propre famille n'était pas exempte. Sous sa plume, les adjectifs jésuitique, italien et fourbe, ont le même sens, et désignaient les courtisans. Ecoutons encore: 'On plume la poule sans la faire crier...on caresse les méchants, on les élève; on humilie les bons, on les écarte.' (PRE 704). Et il conclut: 'jamais le royaume de France ne sera heureux que notre monarque n'aime les roturiers, au lieu d'aimer la noblesse... La noblesse a bien l'air de n'être que les frelons de la ruche qui mangent le miel sans travailler' (PRE 682). Et encore: 'Je dirai que ce que l'on nomme grands courtisans est la perte du roi, comme prince et comme homme, des moeurs, des finances, de la discipline, de tout gouvernement... A quoi donc sont-ils bons? A eux-mêmes seuls... loin de révérer le prince, ils le méprisent et le font mépriser...un fin courtisan est un homme d'une fourberie déliée qui fait le plus de mal à ceux dont il dit le plus de bien, qui poignarde ceux qu'il caresse..., qui fuit les dangers, qui recherche la mollesse, qui sacrifie tout à l'argent..., qui est haut avec les faibles et bas avec les plus puissants que lui, qui s'écarte chaque jour davantage de la franchise gauloise' (PRE 691). Un article des REL intitulé 'De nos courtisans, sources de leurs petitesses, et des vices de leurs coeurs $^{\prime 13}$ cherche à expliquer ce qui rend ces derniers 'si plats et si 
méchants.' Ce sont des courtisans ambitieux qui ont voulu la guerre contre la pragmatique, et, ajoute d'Argenson, ' 1 'on a vu les mauvaises suites' (PRE 693). Les seigneurs de cour sont doublement à craindre, car ils imposent la mode et donnent le ton à tout le peuple: 'il faut dépenser beaucoup pour aimer les pagodes, les belles tabatières et les porcelaines de Saxe, avoir une petite maison, des équipages à la dernière mode, des officiers de la nouvelle cuisine, loger au faubourg St-Germain; en un mot, toutes les choses à charge, peu honorables, de faux plaisirs préférés aux vrais' (PRE 700).

La conclusion à laquelle le marquis est arrivé (même avant sa disgrâce, et qui n'est pas par conséquent le simple fruit de son amertume), s'exprime avec une violence qu' on a l'habitude d'associer à la génération de Figaro plutôt qu'à celle de Voltaire: 'Les grands seigneurs, écrit-il, race à détruire absolument en France. J'entends par grands seigneurs des gens qui ont des dignités, des biens, des titres, des charges, des fonctions, qui, sans mérite, sans être à peine des hommes, n'en sont pas moins grands, et qui par cette raison ne valent nécessairement jamais rien' (PRE 136).

Mais la lecture des textes inédits du marquis révèle, en outre, l'existence d'un personnage qui résumeà lui seul les torts de toute une société. Ce personnage est l'objet d'une étrange lettre de dénonciation rédigée en 1750 et destinée au roi (mais probablement jamais expédiée): 'Que V.M. cherche quel est (le ministre) qui a le plus de crédit et de faveur; $c^{\prime}$ est celui qui la trompe le plus et qui avance davantage les maux du royaume; elle le nommera Elle-même.' Il s'agit du propre frère du marquis, le comte Marc-Pierre d'Argenson. Le Dictionnaire de biographie française pose le problème des rapports entre les deux frères, et conclut qu'ils étaient satisfaisants. Rien n'est plus faux. Dans les archives d'Argenson se trouve un document étonnant de huit pages, de la main du marquis, intitulé 'La vraie et seule cause de ce que j'ai essuyé de disgrâces dans ma vie,' et qui commence ainsi: 'J'ai eu un frère, il m'a envié et haï par envie. Je ne l'ai jamais ni envié ni haï.' Courtisan accompli, le comte devient le symbole de celui qui réussit (le marquis craignait de le voir nommer premier ministre), qui connaît les règles du jeu, qui a du charme, et du goût pour l'intrigue. Le duc de Luynes, dans son portrait du marquis, confirme d'ailleurs ce contraste entre les deux frères: 'Il a moins d'esprit que son frère et d'une autre espèce, écrit-il en parlant du marquis, ... craintif, doutant de lui-même, pensant plus qu'il se communiquait, incapable de composer son visage quand on lui débitait un mensonge, méprisant les détails de l'intrigue, simple et droit, il n'avait rien de ce qu'il fallait pour réussir dans un milieu corrompu.' Le marquis attribue tous ses déboires à une seule cause: la malignité de son frère, aiguisée par l'envie. Le cri vient du coeur: 'Tout jeune je déplus 
à mon père par les soins que mon cadet prit de m'enlever son affection et de s'élever sur mes ruines... Il me fit accabler par une brigue dans l'intendance du Hainaut...Il me mit mal avec le cardinal de Fleury dès qu'il y fut bien. Il me fit injustice sur le traitement de l'ambassade de Portugal... Mon ministère n'a été qu'un cours de panneaux qu'il m'a tendu, de ses créatures qu'il m'a fait prendre pour me perdre... Sa joie a éclaté à ma disgrâce... Les moindres amis que l'on m'a sus n'ont pas été négligés; on me les a enlevés sans savoir comment...enfin il s'attache à ma famille.' Le marquis prétend en effet que son frère a su lui aliéner aussi son fils, son gendre, et sa femme.

Dans un article des REL rédigé un an plus tard, on retrouve les mêmes accusations et le même bilan désastreux: 'm.f. (mon frère) $m$ 'a chassé de toutes les places que j'avais obtenues successivement par travail et par réputation de mérite et de candeur. Il m'a poursuivi avec lâcheté, trahison partout, d'amour couvrant la haine; il m'a enlevé ma fille et le moindre ami. Il a autorisé ma femme contre moi. Je suis dans le néant de tous les emplois publics et de toute considération... Mon frère et ma belle-soeur... regorgent de biens qu'ils ont volé à l'état... J'ai moins de patrimoine que de dettes. Si la cour me retirait mes pensions, si on me les payait mal, je serais sans pain. ${ }^{14}$ Dès décembre 1740 il reprochait au comte ses amis 'qui me déchirent partout... je le sais bien et je sais les conversations; on me reçoit dans le Palais Royal comme un juif ou comme un lépreux.' ${ }^{15}$

Paranoïa ou véritable complot? Le débat est rousseauesque. Autre dilemme, également rousseauesque: faudrait-il taxer le marquis de naïveté politique incurable, ou louer son refus du mensonge quand on lit sous sa plume ce leitmotiv de ses interventions sur la scène publique: 'Quoy qu' on dise, la franchise produira toujours plus quel'habileté dans les affaires politiques'? ${ }^{16}$ Ce qui est sûr, c'est que l'accès au roi était interdit à tout sujet ne sachant pas, ou ne voulant pas, jouer le jeu des courtisans tant détestés par d'Argenson. Toute nouveauté était effectivement étouffée; ce fut après tout pendant ces mêmes années que Voltaire, même avec la protection des deux frères d'Argenson, et dont le goût pour l'intrigue surpassait de loin celui du marquis, essaya sans succès de pénétrer à la cour. A la fin de sa vie, longtemps après son échec politique et celui de son célèbre camarade de classe, d'Argenson désespère de voir réformer la monarchie française ('notre roi est un gouffre de dépenses, écrit-il, et la cour est devenue le sénat de la nation') et il propose à la place le modèle suédois ('un peuple libre sous un roi comme eux'). Il faudrait que les rois de France renoncent au pouvoir arbitraire monarchique, et acceptent une liste civile limitant les dépenses pour la personne du roi, comme c'était déjà le cas en Angleterre, en Prusse et en Suède. Mais le temps des illusions est révolu. 'Ce sont leurs 
valets et leurs putains qui leur conseillent le contraire, ${ }^{17}$ mais cette fois-ci, en août 1756, on sent que le marquis est prêt à admettre qu'encore une fois ce sont justement ceux-là qui ont gagné la partie.

\section{NEAL JOHNSON \\ University of Guelph}

\section{Notes}

1 V. par exemple PDP 6: 'L'égalité est la perfection. L'aristocratie est à la démocratie ce que la pourriture est au fruit'; PDP 9: 'l'hérédité de la noblesse est un faux principe... quand je parle ainsi, l'on ne m'écoute plus; on me regarde en France comme un homme à idées singulières. On baille.'; PDP 17: 'Quoique je sois aîné de ma maison, je déclare ici que si j'étais chargé de la réforme de nos lois, j'abolirais presque tout droit d'aînesse.'

2 'On ne conçoit pas comment un seul homme a tant travaillé; ce qu'il a lu, écrit de sa main, critiqué, pensé, est prodigieux.' REL 1831.

3 Misère du royaume, harangue dans le goût anglais, mémoire du 23 août, 1747.

4 V. REL 1106, 'Histoire d'un nigaud'; aussi Journal (éd. Rathery) III, 116 et V, 347.

5 PDP art. 53, sept., 1751.

6 Remontrance au roi sur le maniement et l'autorité de toutes ses affaires qui sont aujourd'hui ès mains des deux frères Pâris, le 26 décembre, 1748.

7 Journal, éd. Rathery, IX, 122. Après la disgrâce du marquis, le roi représentait en même temps son seul espoir de reprendre un jour le pouvoir. Dans des rêves aussi pathétiques qu'irréels il pouvait écrire 'j'ai imaginé quelquefois...que Sa Majesté...me tirerait dans le particulier pour me dire qu'elle n'avait vu que moi de bonne foi dans tout ce qui se mêle de ses affaires, qu'Elle voulait révoquer les ministres intrigants et qui la trompaient, et qu'Elle était dans le dessein de m'établir premier ministre.' PRE 697, sept. 1747.

\section{V. PRE 702.}

9 PDP art. 52, juin, 1752.

10 lettre du 27 juillet, 1747.

11 Avis à S.M., fév., 1748.

12 Lettre au roi, juin, 1750.

13 REL 1134.

14 REL 1207.

15 lettre du 11 déc., 1740 .

16 lettre du 24 juin, 1747.

17 REL 2496, août, 1756. 